Indian Journal of Clinical Biochemistry, 2002, 17 (2) 96-100

\title{
CLINICAL ASPECTS OF TUBERCULOSIS
}

\author{
V.K. Vijayan \\ Director, VP Chest Institute, University of Delhi, Delhi-110007
}

Pulmonary tuberculosis is a chronic infectious disease caused by Mycobacterium tuberculosis (1). Other mycobacteria can also produce pulmonary tuberculosis and these include Mycobacterium africanum, and Mycobacterium bovis. Usually, patients with pulmonary tuberculosis who have cavitary lesions are an important source of infection. These patients are sputum smear-positive. Coughing produces tiny infectious droplets. Usually, one bout of cough produces 3000 droplet nuclei and these can stay in the air for a long period of time. Ventilation removes these infectious nuclei. Mycobacterium tuberculosis can survive in the dark for several hours. Direct exposure to sun light quickly kills these bacilli. Of the various factors, determining an individual's risk of exposure, two factors are important. These include the concentration of droplet nuclei in contaminated air and the length of time that air is breathed. The risk of transmission of infection from a person with sputum, smear-negative pulmonary tuberculosis and miliary tuberculosis is low and with extrapulmonary tuberculosis infection is even lower. However, infection with Mycobacterium bovis occurs through milk. Human infection from Mycobacterium bovis is rare in India because milk is often boiled before use. Even though non-tuberculosis mycobacteria are harmless, some can cause human disease especially in immunocompromised individuals (2)

The cardinal event in the pathogenesis of tuberculosis, whether inapparent or overt is the implantation of Mycobacterium tuberculosis in the tissues. Lung is the most frequent portal of entry. The organism enters the lung from the inhalation of air borne droplets which have been coughed out by 'open' [sputum-positive] pulmonary tuberculosis patients who have received no treatment, or have not been treated fully. Droplet nuclei are airborne particles of water, usually 1 to 4 micrometers in diameter, in which the bacilli travels. Deposition of

Author for Correspondence :

Dr. V.K. Vijayan

Director, V.P. Chest Institute, University of Delhi

Delhi-110 007

Phone: 7667180 , Telefax: 7667420

E-mail : <vijayanvk@hotmail.com

Indian Joumal of Clinical Biochemistry, 2002 bacilli occurs in the alveoli by sedimentation. Gravitational forces in the peripheral lung regions where airflow velocity is low aid in the sedimentation of bacilli. This elicits a local inflammatory response with neutrophils and resident macrophages during the first 48 hours. The organisms proliferate in the alveoli at the periphery of the lung, often beneath the pleura forming a Ghon's focus. Acute inflammatory response that occurs at the site of implantation may result in some cell death. As immune response has not developed, the organism is not destroyed and they are conveyed to regional lymph nodes at hilum resulting in proliferation of bacteria in the lymph nodes. Immune response develops 4 to 6 weeks after inhalation and activated $T$ cells recruit monocytes and mononuclear cells to the lung and lymph nodes, ultimately leading to granulomatous inflammation with giant cells, epitheloid cells and lymphocytes and caseation. Usually the Ghon's focus and caseating granuloma in lymph nodes heal by collagen deposition around the tubercle. The lesion at the primary site of involvement, draining lymphatics and the inflammed regional lymph nodes constitute the primary complex. When the primary site of implantation is in the lung, it is called Ghon's focus. The draining lymphatics and the involved lymph nodes together with Ghon's focus constitute the primary complex [Ghon complex]. In children, the lymph node component may be much larger than the Ghon's focus. Viable bacteria may remain walled off within the healed primary complex leading to latent tuberculosis. Progressive primary tuberculosis results in individuals who are unable to mount an appropriate immune response. Spread of infection can occur when lymph nodes erode through the wall of a bronchus or a blood vessel. Ghon focus, though small, can rupture through the visceral pleura to produce tuberculous pleurisy. In $75 \%$ cases, it occurs within 6 months of infection. Bronchial spread of the organisms may result in extensive confluent granulomatous lesions and this may be fatal. Spread of the organisms through blood stream results in miliary tuberculosis. If the lymph nodes erode into a pulmonary vein, this results in systemic dissemination of the organisms. If node ruptures into a pulmonary artery, miliary dissemination to 
the lungs occur. Less commonly, tubercle bacilli may be ingested and lodge in the tonsil or in the wall of the intestine. This form of tuberculosis occurs following the ingestion of contaminated milk or milk products. Rarely, tuberculosis can occur as a result of direct implantation of the organisms into the skin through cuts and abrasions. This form of tuberculosis is a health hazard faced by health care workers and laboratory staff who handle materials infected with Mycobacterium tuberculosis. These lesions were termed "prosector's warts" (2). Interestingly, Laennec, the inventor of the stethoscope, acquired tuberculosis in this fashion which eventually led to his death (2).

Thus, primary tuberculosis is a widely disseminated infection. This fact is not realized by clinicians. Most of the metastatic foci heal. However, some of these metastatic foci may remain dormant and may reactivate at a later date when the host resistance decreases (3). The subsequent course of the events varies considerably. In most of the patients, the primary complex resolves without becoming clinically apparent. This occurs when the immune status of the host is good, and healing occurs by fibrosis and calcification. In a minority of patients, progressive primary tuberculosis due to the extension of the inflammatory process at the site of the primary focus can occur(4). In the lung, this can present as an area of consolidation [tuberculosis pneumonia]. This form of the disease was often encountered in the prechemotherapeutic era and was termed "galloping consumption" or "pneumonia alba" [white pneumonia]. This form is encountered in the present era in patients with human immunodeficiency virus (HIV) infection: Caseation necrosis at the Ghon's focus may lead to liquefaction. Expectoration of the liquefied material can leave a cavity with shaggy margins in the pulmonary parenchyma which may be apparent on the chest radiograph.

Mediastinal and tracheobronchial lymph nodes may produce compression of the adjacent bronchus. If the obstruction is complete, the lung distal to the site of bronchial obstruction becomes atelectatic. If the obstruction is incomplete, it may act as a "ballvalve" and results in obstructive emphysema. The inflamed caseous lymph nodes may erode through the walls of the bronchus and result in bronchogenic dissemination. Bronchial mucosal involvement may result in tuberculosis bronchitis. In a patient with overwhelming infection, large number of

Indian Journal of Clinical Biochemistry, 2002
Mycobacterium tuberculosis may gain access to the circulation and result in miliary and meningeal tuberculosis. In majority of the patients, the initial focus of infection subsides. Cicatrisation, scar formation and often calcification develop. Repeated episodes of extension of infection followed by healing and fibrosis may result in the formation of "onion skin" or "coin lesion" (2).

Tuberculosis infection refers to a positive tuberculin test without evidence of active disease. Tuberculosis disease refers to active disease with positive acid fast smear or culture for $M$. tuberculosis or radiographic and clinical presentations of tuberculosis.

Diagnostic tools that are available today for the rapid diagnosis of tuberculosis have remained largely the same since the discovery of tubercle bacillus by Robert Koch more than a century ago. The main diagnostic tools are :

$\begin{array}{ll}1 & \text { Clinical characteristics, } \\ 2 & \text { Tuberculin skin testing, } \\ 3 & \text { Chest radiography, } \\ 4 & \text { Sputum smear microscopy, } \\ 5 & \text { Culture of bacteria, } \\ 6 & \text { Serology and antigen detection and } \\ 7 & \text { Molecular diagnostic methods. }\end{array}$

\section{Clinical Characteristics}

A high clinical index of suspicion is the most important factor in the early diagnosis of tuberculosis especially in primary tuberculosis. Clinicians should be aware of the groups in which tuberculosis is more common. These include patients with HIV infection, the elderly, persons born or residing in places where infection with tuberculosis is common, close contacts of known cases of active tuberculosis, persons with malignancy, malnutrition, or diabetes mellitus, and persons receiving immunosuppressive therapy or underlying lung diseases such as silicosis(5).

In vast majority of cases, primary infection is asymptomatic or minimally symptomatic with fever, dry or scant sputum production. Occasionally patient may develop retrosternal pain or erythema nodosum. Primary infection can cause clinical pneumonia in 5 to $10 \%$ adults. This percentage may be higher in children and HIV infected persons. In older infants and children, primary pulmonary 
tuberculosis usually produces no signs or symptoms and a chest $X$-ray shows no signs of infection. Rarely, there may be enlargement of lymph nodes, and perhaps some coughing. In most cases tuberculin skin test is positive. This primary. infection usually resolves on its own as the child develops immunity over a six to ten week period. But in some cases, it can progress and spread all over the lungs (progressive tuberculosis) or to other organs. This causes systemic signs and symptoms such as fever, weight loss, fatigue and loss of appetite, and signs and symptom referable to the organ involved.

It has been estimated that approximately $10 \%$ of individuals with normal immunity will develop active TB within their life time, and $5 \%$ within the first two years of infection. The progression from TB infection to disease is related to host factors such as genetics, nutritional status and immune competence. The incidence of TB in HIV-infected persons is more than 100 times that of general population. The risk of TB developing in an HIVinfected person who is latently co-infected with Mycobacterium tuberculosis is approximately $10 \%$ per year.

Tuberculosis should be suspected in patients presenting with the following symptoms and history:

\section{Adults:}

1 Cough more than three weeks.

2 Blood in the sputum,

3 Chest pain for more than one month.

$4 \quad$ Increasing weakness and loss of weight and

5 Had tuberculosis in the past or previously treated for cough?

\section{Children:}

1 Close contact with a smear-positive case of tuberculosis.

2 Positive tuberculin test.

3 Wasting: decrease in weight with no obvious reasons and

$4 \quad$ Two or more episodes of fever with no obvious cause such as malaria.

However, the clinical characteristics are non-specific and these symptoms point to do further investigations to arrive at a firm diagnosis.

\section{Tuberculin Skin Testing:}

Even though a positive response to tuberculin which has been described more than 100 years ago is not diagnostic of tuberculosis, it significantly increases the suspicion of tuberculosis (6). It is a simple, cheap and easy to read test. Tuberculin test is performed using PPD-S or PPD-RT23. The strength of PPD is expressed in terms of International Units (IU). One unit of PPD-RT 23 corresponds to about three IU of PPD-S. For diagnostic purpose, the test is performed with 5 IU PPD-S or one or two units of PPD-RT 23+ Tween 80 . Tween 80 is used as a detergent to prevent the tuberculin sticking to the glass. Tuberculin test is performed by injecting one unit of PPD-RT 23+ Tween 80 intradermally producing a lump in the skin of 5-6 $\mathrm{mm}$ diameter The skin test reaction is read 48 to 72 hours later( 7 ). If positive, an area of erythema and an area of induration of the skin is seen. The diameter of the Induration is measured across the transverse axis of the arm. A positive reaction is a diameter of 10 $\mathrm{mm}$ or more with one PPD-RT23 in subjects who has not been vaccinated with BCG. If a child is vaccinated with BCG, a diameter of over $15 \mathrm{~mm}$ is recognized as positive, suggesting that this child has also been infected with M.tuberculosis. A positive result means that the person is infected by M. tuberculosis, but it does not mean that he/she has tuberculous disease. Similarly, a negative test means that the person has not been infected with $M$. tuberculosis. However, it does not exclude infections with $M$. tuberculosis. A number of factors can suppress tuberculin reactions e.g. malnutrition. HIV infection, measles, chicken pox, cancer, severe bacterial infections (including tuberculosis) and corticosteroids. It may also be negative, if tuberculin test is performed in a person soon after inhaling the organısm. It does not differentiate primary and secondary infections, and active and inactive diseases

\section{Chest Radiography}

Chest radiography is widely used for detecting cases of pulmonary tuberculosis. However, no radiographic picture is typical of tuberculosis. and many lung diseases can have sımılar radıographic appearances mimicking pulmonary tuberculosis. It cannot distinguish active pulmonary tuberculosis from inactive and previously treated tuberculosis. In 
addition, chest radiography may not detect early stages of tuberculous disease. Because many a time chest radiography is used as a means for the diagnosis of pulmonary tuberculosis by many physicians, it can lead to over-diagnosis of TB and unnecessary drug treatment. It has also been observed that. even among expert readers, on an average there was $25 \%$ of under-reading and about $2 \%$ of over-reading. It was also found that there was an intra-individual inconsistency of $20 \%$, i.e. each reader changed his/her mind in about one-fifth of the cases helshe had classified as "positive" at the first reading (8). Even though chest radiography is not a reliable test for the diagnosis of pulmonary tuberculosis, it can be a useful supportive tool in the diagnosis of primary and smear negative pulmonary tuberculosis

In primary tuberculosis, a lobar or segmental infiltrate characterized by ipsilateral hilar adenopathy is seen in chest radiographs. Hilar adenopathy may lead to compression of the bronchus and displacement of the great vessels and trachea. Resolution of primary tuberculosis may lead to a parenchymal nodule or Ghon's focus which becomes calcified with time. When a Ghon's focus is associated with calcified hilar lymph nodes, it is termed as primary or Ranke's complex. Primary tuberculosis infection may progress in a small percentage of patients resulting in pleurisy and pleural effusion, progressive caseous pneumonia, extensive broncho-pneumonia or hematogenous spread that leads to disseminated disease.

Computed Tomography (CT) is more sensitive than chest radiography for detection of lymphadenopathy, miliary disease, bronchiectasis. bronchial stenosis. broncho-pleural fistula and pleural effusions. CT may reveal occult abscesses and the extent of pleural disease. Magnetic resonance imaging is useful in extrapulmonary disease, such as skeletal and intracranial tuberculosis

\section{Sputum Smear Microscopy}

Detection of acid fast bacilli in clinical specimens described by Robert Koch more than a century ago, is still the most important diagnostic test in tuberculosis. It is cheaper, easier and more reliable than chest radiographs and tuberculin testing, and cheaper and easier than culturing of Mycobacterium tuberculosis. Even though acid fast bacilli(AFB) stain of sputum is positive in upto $75 \%$ of adults with pulmonary TB, fewer than $20 \%$ of children with TB Indian Journal of Clinical Biochemistry, 2002 have a positive AFB smear of sputum or gastric aspirate(9). Fluorochrome stains such as auramine and rhodamine are superior to classical carbol fuchsin stains. The lower limit of detection of AFB sputum smear is of $0.5-1 \times 10^{4}$ micro-organisms $\mathrm{mL}^{-1}$ of sample. AFB smears cannot distinguish M.tuberculosis from other mycobacteria. It also cannot distinguish dead organisms from live ones.

\section{Culture of Mycobacteria}

Isolation of $M$. tuberculosis in culture from clinical specimens is the gold standard for the diagnosis of tuberculosis(10). Culturing is more sensitive than sputum smear microscopy. But it is an expensive method requiring trained personal. It takes 6-8 weeks to obtain culture results in conventional solid media and this delays confirmation of the diagnosis and starting treatment. Culturing is therefore inappropriate as a diagnostic tool, but can be used to test drug susceptibility. Culturing is also useful in sputum smear-negative cases where active TB is suspected. There should be a minimum of 100 mycobacteria per $\mathrm{ml}$ for a positive culture. In routine laboratories, a large proportion of positive cultures are due to contamination. BACTEC technique is a semi-automated radiometric liquid culture system to detect the growth of mycobacteria more quickly than is possible with conventional solid media(11). BACTEC cultures may become positive as soon as $1-2$ weeks after inoculation. However, it is an expensive system. With the Septi-Check technique, the rate of recovery is higher than that with conventional cultures and with BACTEC technique(12).

Currently. serological investigations have no value in the diagnosis of tuberculosis. The polymerase chain reaction (PCR) is a technique that allows the rapid detection and quantification of few DNA copies with very high sensitivity and specificity. Diagnostic PCR involves DNA amplification using DNA sequences as markers for microorganisms (13).

\section{Treatment}

Treatment of tuberculosis is by using appropriate regimens of six or eight month duration. Sputum smear-positive new pulmonary tuberculosis patients are treated with a six month regimen that includes the first two months of intensive phase with isoniazid(H], rifampicin[R], ethambutol[E] and pyrazinamide[Z] followed by the next four months with isoniazid and rifampicin. Patients with relapse and resistant form of tuberculosis can be treated with a eight month regimen that includes three 
months intensive phase with HREZ(during the first two months of intensive phase, streptomycin is also given) followed by next five month s with HRE

Sputum smear negative tuberculosis is treated with a six month regimen. Initial intensive phase of two months is with HRZ followed by the next four months with HR. In all regimens the drugs can be given either daily or three times a week.

\section{REFERENCES}

1. Koch R. The aetiology of tuberculosis ( $A$ translation by Pinner $B$ and Pinner $M$ from the Berl in Kliniche Wochenschrift, 1882; XIX: 221). Buil Int Union Tuberc 1981, 56:87-100

2. Grange JM. Mycobacteria and human disease. London: Arnold; 1996.

3. Vijayan VK. Pulmonary tuberculosis. In: Tuberculosis Eds. SK Sharma and A Mohan, Jaypee, New Delhi, 2001:152-158.

4. Udani PM and Saroj Mehta. Tuberculosis in children. In: Tuberculosis. Eds. SK Sharma and A. Mohan, Jaypee, New Delhi, 2001; 413-425

5. Miller FJW. Tuberculosis in children. Edinburgh. Churchill Livingtone. 1982. Schulger NW and Rom WN Current approaches to the diagnosis of active pulmonary tuberculosis. Am J Respir Crit Care Med 1994; 149:264-267.

6. Koch R. Weitere Mitteilungen uber ein Heilmittle gegen Tubercuose, Dtsch Med Wschr 1891;17:101102.

7. Crofton J, Horne N and Miller R. Mantoux test. In: Clinical Tuberculosis. CBS Publications \& Distributors. Daryaganj, New Delhi 1996;188-190.

8. Toman K. Case Finding. In: Tuberculosis. Case-finding and chemotherapy: Questions and Answers. World Health Organisation, Geneva 1979:3-72.

9. Lipsky BA, Bates J, Tenover FC, Plorde JJ. Factors affecting the clinical value of microscopy for acid-fast bacilli. Rev Infect Dis 1984:6:214-22.

10. Fregnan GB, Smith DW. Description of various colony forms on mycobacteria. J Bacteriol 1962:83:819827.

11. Siddique $\mathrm{SH}$, Libonati JP, Middlebrook $\mathrm{G}$. Evaluation of a rapid radiometric method of drug susceptibility testing of Mycobacterium tuberculosis 1981;13:908-12

12. D'Amato RF, Isemberg HD, Hochstein L, Mastellone AJ, Alperstein P. Evaluation of the Roche SeptiCheck AFB system for recovery of Mycobacteria. J Clin Microbiol 1993:31:2689-2691.

13. Catanzaro A. Rapid diagnostic tests for tuberculosis: A major breakthrough coupled with major responsibilities. Pulmonary Perspectives. American College of Chest Physicians. 1998;15:2-5. 